\title{
Modelo de universidad comunitaria intercultural y su aporte a las economías comunitarias en contextos multiculturales
}

\section{The Intercultural community university model and its contribution to community economies in multicultural contexts}

Angélica Leonor Ruiz Calderón'

\section{Resumen}

El modelo comunitario intercultural de la Universidad de las Regiones Autónomas de la Costa Caribe Nicaragüense (URACCAN), es un proyecto educativo que trabaja con los distintos pueblos y comunidades étnicas que conviven en el diverso territorio de Nicaragua; tiene como propósito formar hombres y mujeres para el fortalecimiento de los pueblos, acompañando procesos sociales e incidiendo en la construcción de sus identidades desde contextos multiculturales. Este tipo de investigación es cualitativo con nivel de profundidad exploratorio.

La URACCAN trabaja con las familias, hijos, hijas de productores y productoras para fortalecer la economía familiar y comunitaria, desde un enfoque socio cultural, basado en la equidad, justicia, reciprocidad y sostenibilidad, son principios que se complementan y se practican como resultado de un acuerdo colectivo e igualitario entre los miembros de la comunidad.

Desde estos espacios se construyen sendas que posibilitan rescatar prácticas educativas y revitalizar los saberes y conocimientos comunitarios que permiten desarrollar pensamientos para el cambio, y que posterior, se traducen para el modelo, en prácticas para fomentar aprendizajes basados en la vida y el desarrollo con identidad en contextos de diversidad de pueblos indígenas, afrodescendientes y mestizos para la promoción de ciudadanías interculturales.

Palabras clave: Educación Superior; economía comunitaria; saberes.

\section{Abstract}

The intercultural community model of the University of the Autonomous Regions of the Nicaraguan Caribbean Coast (URACCAN) is an educational project that works with the different peoples and ethnic communities that coexist in the diverse territory of Nicaragua; Its purpose is to train men and women to strengthen peoples, accompanying social processes and influencing the construction of their identities from multicultural contexts. This type of research is qualitative with a level of exploratory depth.

URACCAN works with the families, sons and daughters of producers to strengthen the family and community economy, from a socio-cultural approach, based on equity, justice, reciprocity and sustainability, they are principles that complement each other and are practiced as a result of a collective and equal agreement among the members of the community.

\footnotetext{
1 Doctora en Educación con especialidad en Mediación Pedagógica, Vicerrectora de la Universidad de las Regiones Autónomas de la Costa Caribe Nicaragüense - Recinto Universitario Las Minas. Correo: vicerectoria.lasminas@uraccan.edu.ni. http://orcid.org/0000-0002-4295-5919
} 
The community economy is presented as a reality in which new and old economic practices are configured which in turn operate as alternative strategies for poverty reduction. It is conceived from ancestral cultures, it is directly linked to our mother nature and is in accordance with the norms of ancestral worldviews, principles and values as the basis of cultures.

From these spaces, paths are built that make it possible to rescue educational practices and revitalize the knowings and community knowledge that allow the development of thoughts for change, and that later, the thoughts are translated for the model, into practices to promote learning based on life and development with identity in contexts of diversity of indigenous, Afro-descendants and mestizos peoples for the promotion of intercultural citizenship.

Keywords: Higher Education; Community Economy; Knowings

\section{Introducción}

La Universidad de las Regiones Autónomas de la Costa Caribe Nicaragüense (URACCAN) nace de los procesos de Autonomía Regional por sostener una educación superior intercultural, pertinente, de calidad, inclusiva y respetuosa de los derechos humanos de mujeres y hombres de los pueblos indígenas, afrodescendientes y étnicos de Abya Yala. Orienta sus esfuerzos hacia la construcción y reconstrucción de conocimientos apoyados en las prácticas y saberes endógenos, aportando al desarrollo con identidad de la región desde la cosmovisión de cada pueblo.

Las economías campesinas y la integración de estrategias familiares han visibilizado las prácticas de las economías comunitarias, han permitido la supervivencia de este sector rural, generando cambios sociales y económicos. El resultado de este modelo económico ha sido la implementación de emprendimientos, cooperativas, espacios de recreación y culturales, desarrollo de servicios y bienes comunitarios.

Este modelo económico, persigue un crecimiento económico con equidad, sostenibilidad ambiental y oportunidad para crecer, para transformar las reglas del juego económico. Pretende una transformación social que permite afirmar la condición de la vida humana, regida por valores de cooperación y compromiso social.

\section{De acuerdo con Leonardo Boff:}

Nuestra percepción de fondo ha cambiado: somos todos interdependientes, habitamos juntos la misma Casa Común, la Tierra. Nadie tiene un futuro particular y propio. Surge un destino común globalizado: o cuidamos de la humanidad [y de la economía humana] para que no se bifurque entre los que comen y los que no comen, y protegemos el planeta Tierra [nuestra casa] para que no sea destruido por el calentamiento global, o no tendremos ningún futuro. Estamos vinculados definitivamente unos a otros (Boff, 2010).

Este modelo de economía como un enfoque local es propio de los pueblos, proyecta su identidad y necesidad para la transformación comunitaria de manera inclusiva. Endógena y abierta al mismo tiempo, justa en el intercambio comercial, generadora de riqueza que promueva el progreso de manera equitativa entre el ser humano y la madre tierra (Valdivia, 2006)

La URACCAN mantiene el principio de la relación teoría y práctica en los procesos educativos, lo que permite integrar la docencia con la actividad investigativa y de extensión comunitaria, de esta manera permite relacionar al protagonista en proceso de formación, con la realidad de su entorno y de sus semejantes, los hace sensible a las necesidades sociales y les permite desarrollar el sentido del emprendimiento y la innovación en la solución a problemas de la sociedad y para su autodesarrollo.

La oferta académica asegura la democratización de la educación, reduciendo las barreras económicas, lingüísticas, de género y generacionales, tecnológicas y culturales que han impedido la accesibilidad a 
procesos y programas de formación en diferentes niveles, fundamentalmente a la Educación Superior de parte de los pueblos indígenas, afrodescendientes y comunidades campesinas mestizas. Hemos venido formando líderes y lideresas, a través de diplomados, talleres, capacitaciones y cursos para el fortalecimiento del liderazgo comunitario, desde un contexto multicultural.

Desde estos espacios se construyen sendas que posibilitan rescatar prácticas educativas y revitalizar los saberes y conocimientos comunitarios que permiten desarrollar pensamientos para el cambio, y que posterior, se traducen para el modelo, en prácticas para fomentar aprendizajes basados en la vida y el desarrollo con identidad en contextos de diversidad de pueblos indígenas, afrodescendientes y mestizos para la promoción de ciudadanías interculturales.

\section{Filosofía institucional y las economías comunitarias}

Desde el modelo pedagógico de la URACCAN, se define que, la educación comunitaria debe ser realista y práctica, reflejar la riqueza espiritual de las cosmogonías y de las filosofías comunitarias como fuentes de sabiduría y de equilibrio armónico del ser humano con la tierra. Debe ser estructurada sobre la transmisión de sistemas integrados de conocimiento holístico y fundamentarse en la espiritualidad.

Los principios son: (i) Ser intercultural, respetuosa y promotora de la convivencia de personas y conocimientos entre culturas; (ii) Responder a las necesidades y particularidades propias de las comunidades; (iii) Que llegue a las comunidades y lugares donde están las personas que toman las decisiones locales, donde están la niñez, la juventud, los hombres y mujeres de las comunidades; (iv) Promover un modelo de gestión descentralizado y participativo; (v) Contribuir a la sostenibilidad de los procesos educativos; (vi) Acompañar procesos de autogestión y autodesarrollo con las comunidades permitiéndoles conocer otras prácticas que enriquezcan su visión del mundo, pero a la vez asegurando que incrementen su capacidad propositiva y de incidencia; (vii) Relacionar lo endógeno con lo exógeno en los procesos educativos, productivos y culturales, logrando con esto conocer y valorar lo externo; pero, sobre todo, valorar y mejorar lo propio; (viii) Que asegure la participación activa de las comunidades y sus autoridades; y (ix) Que fomente la interculturalidad en los procesos educativos, como un espacio de interrelación de confianza y esperanza entre los sujetos.

El desarrollo integral y sustentable de las Regiones Autónomas y de cualquier otro lugar, descansa en las capacidades, el talento, la visión, la ardua labor y el compromiso inquebrantable del talento humano, en sus diferentes niveles de división del trabajo (individual y colectivo), para construir una vida digna y el pleno ejercicio de sus derechos humanos. Esto, en gran medida, mediante la creación de oportunidades para la generación y distribución equitativa de la riqueza (incluyendo iniciativas encaminadas a aumentar la productividad, el nivel de ingresos, la calidad del empleo y la protección social en el trabajo), con la inclusión en este proceso de los segmentos menos favorecidos (Zuniga,2013).

\section{Aportes, experiencias de economías comunitarias}

La URACCAN desde su modelo de Educación Superior Comunitaria e Intercultural en la Costa Caribe nicaragüense permite el acceso a la formación de mujeres y hombres de los pueblos indígenas, afrodescendientes y mestizos de la región y del país. Los niveles de formación que oferta la universidad son: educación de grado, postgrado y educación continua. Todos los procesos de formación son producto de las demandas sociales, políticas y programas de desarrollo de la región y del país, por tanto, la educación se convierte en el proceso principal para el fortalecimiento de los procesos autonómicos de los pueblos indígenas, afrodescendientes y comunidades étnicas de la región (Hooker, 2018).

Las funciones estratégicas de la universidad desde su Modelo Comunitario Intercultural se orientan en la comunidad del aprendizaje: investigación, acompañamiento comunitario, la gestión y la cooperación externa, siendo la formación un elemento inherente al desarrollo de las comunidades y de las familias. A pesar de que existen espacios de formación de parte de otras instituciones educativas, las ofertas académicas no 
generan oportunidades para asegurar una formación a corto plazo para los jóvenes que permita potencializar sus fincas y convertirlas en sus propias empresas. El acceso a la educación de los jóvenes comunitarios se traduce en anhelos y derecho de la educación de calidad.

Las experiencias realizadas por la URACCAN en la formación de jóvenes de campo en comunidades rurales han dado oportunidades para contribuir en el desarrollo económico de las familias rurales a nivel municipal, así como en las cadenas de valor de cacao, café y ganadería que son las bases de las economías de los pueblos en esta región, dando solución a los problemas que demanda la sociedad costeña y por ende a las demandas de las agendas de vida y planes de desarrollo que los pueblos establecen para la sostenibilidad de sus economías.

En este esfuerzo, se toma en cuenta como célula fundamental de la sociedad a la familia que, constituye un importante eslabón en la formación y conocimientos de todos sus miembros, conduce a fomentar una tradición en valores que, finalmente es cosecha del tejido social que se plantea en las dinámicas comunitarias, el que se traduce en bienestar y buen vivir para el fortalecimiento de las autonomías de los pueblos.

Desde la concepción de la economía comunitaria, los medios de trabajo y los recursos naturales son de propiedad comunal y / o colectiva, donde los miembros de la comunidad son los propietarios de los recursos existente dentro del territorio, trabajados en forma privada y familiar.

Desde esta experiencia de las economías comunitarias, se busca mantener su esencia, símbolos, principios, generando sentido de pertenencia de un todo, donde cada miembro que participa en la producción, también lo en la organización y en la toma de decisiones. No genera desigualdades hacia el interior de la comunidad y entre comunidades dentro de un territorio y espacio, organizándose en grupos familiares.

La economía comunitaria busca el bienestar común, de la comunidad y no sólo individual, esta economía esta generada, producida, gestionada por la propia comunidad. Además, es concebida desde las culturas ancestrales, desde el ser humano y su relación con la madre tierra y va acorde a las normas de las cosmovisiones ancestrales, principios y valores de todas las cosmovisiones de los pueblos originarios, afrodescendientes y mestizos.

En este sentido la economía comunitaria viene a ser también integral como las culturas y está orientada no al beneficio individual, sino más bien al beneficio común, donde no está negado el individuo, ni los grupos, sino que se articula con lo que está dentro de la comunidad, integra y realiza todo ello.

\section{La economía comunitaria y los principios de la ODS}

La economía comunitaria, además de ser un elemento que caracteriza las culturas y cosmovisiones de los pueblos, desde el respeto de las prácticas ancestrales es una alternativa climáticamente inteligente y resiliente, que armoniza la producción primaria, basada en el aprovechamiento de los recursos naturales, de forma sostenible con la madre tierra, pero productiva, tomando en cuenta el conocimiento científico, técnico, local y ancestral; en la práctica de la agricultura, ganadería, forestal, pesca, agroindustria y en este contexto el turismo y otras estrategias para la generación de ingresos.

Además, promueve la disponibilidad, el consumo, el acceso, la estabilidad y la inocuidad de alimentos nutritivos, esto a largo plazo apunta hacia la sostenibilidad de los medios de vida desde una perspectiva integral. Tomando en cuenta la transformación, el comercio en el ambiente local encaminado a implementar acciones en toda la cadena de valor de los rubros y sectores en el territorio.

\section{Conclusión}

La economía comunitaria constituye un modelo de desarrollo que, comprende sistemas de planificación, organización, producción, generación de excedente y su distribución para el bienestar común; basado en 
la cosmovisión de los pueblos indígenas, originarios y campesinos, en comunidades interculturales y afrodescendientes quienes administran su territorio, recursos y tienen sus propias formas de organización en armonía y equilibrio con la madre tierra.

En la economía comunitaria, el sistema socioeconómico está basado en la solidaridad, la reciprocidad y la redistribución.

Las economías comunitarias aportan al desarrollo y mejora las condiciones de vida de las familias en la región, esto debe tomarse como un referente para la toma de decisiones en la promoción de planes de vida comunitarios y territoriales.

\section{Recomendaciones}

La dinámica económica de las Regiones Autónomas y las condiciones de productividad y competitividad de sus agentes económicas están condicionadas por el desarrollo de las fuerzas productivas. Para ello es necesario disponer no sólo del talento humano, sino que brinde información sobre las condiciones socioeconómicas de su población y su relación con el mundo laboral, el desarrollo y el acceso a los mercados y los desafíos de orden macro y meso que deben enfrentar para orar un desarrollo productivo y económico sostenible.

Como universidad desde el modelo de comunitaria e intercultural es importante incorporar el estudio y promoción de las economías comunitarias en los planes de docencia, investigación y extensión para generar conocimientos acerca de sus características, dinámicas e interacciones entre los pueblos, a fin de promover su revitalización, el buen vivir y la construcción de ciudadanías interculturales.

\section{Agradecimiento}

Esta publicación obtuvo el financiamiento de: El Fondo de Asistencia Internacional de los Estudiantes y Académicos Noruegos (SAIH).

\section{Lista de referencias}

Boff, Leonardo, (2010). Una ética de la madre tierra. Como cuidar la casa común. Editorial Trotta

Hooker, A. (2018). Educación Superior pertinente y de calidad. Inclusión, diversidad, derechos humanos y extensión social: Experiencia de la Universidad Comunitaria Intercultural. CARIBE, 23-27.

Zuniga, Morales V. (2013). Evaluación interna del proyecto Fortalecimiento de URACCAN Las Minas, Fase IV.

Valdivia, María Paz. (2006). "Cosmovisión Aymara y su Aplicación Práctica en un Contexto Sanitario del Norte de Chile”, Revista de Bioética y Derecho $N^{\circ} 7$, 